\title{
On iterates of $\boldsymbol{e}^{z}$
}

MICHAK MISIUREWICZ $\dagger$

Department of Mathematics, University of Maryland, USA; and Mathematics Institute, Warsaw University, Poland

(Received 8 November 1980)

Abstract. It is proved that for the map $f: \mathbb{C} \rightarrow \mathbb{C}$ given by $f(z)=e^{z}$, the family $\left\{f^{n}\right\}_{n=0}^{\infty}$ is not normal at any point. In particular, $f$ is topologically transitive.

Let $f: \mathbb{C} \rightarrow \mathbb{C}$ be an analytic function. In the theory of iterates of $f$ an important role is played by the set $F(f)$ of points at which $\left\{f^{n}\right\}_{n=0}^{\infty}$ is not a normal family.

The aim of this paper is to prove that, for the map $f(z)=e^{z}$, the set $F(f)$ is the whole plane. This was conjectured in 1926 by Fatou [2]. Some progress has been made by Töpfer [3] who proved that $F(f) \supset \bigcup_{n=0}^{\infty} f^{-n} \mathbb{R}$, and by Baker [1] who proved that any limit function of a subsequence of $\left(f^{n}\right)_{n=0}^{\infty}$ on any component of $\mathbb{C} \backslash F(f)$ has to be one of the constants $f^{n}(0), n=0,1, \ldots,+\infty$. In my proof I do not use the above results.

More information concerning this and similar problems, together with historical references, can be found in [1].

In the sequel we assume that $f(z)=e^{z}$.

LemMA 1. Let $z \in \mathbb{C}$. Then $\left|\operatorname{Im}\left(f^{n}(z)\right)\right| \leq\left|\left(f^{n}\right)^{\prime}(z)\right|$.

Proof. We have $f(x+i y)=e^{x} \cos y+i e^{x} \sin y$. Since $|\sin y| \leq|y|$, we obtain $|\operatorname{Im}(f(w))| \leq|\operatorname{Im} w| \cdot|f(w)|$ for every $w \in \mathbb{C}$. But $f^{\prime}(w)=f(w)$, and hence if $w \notin \mathbb{R}$ then $|\operatorname{Im}(f(w))| /|\operatorname{Im} w| \leq\left|f^{\prime}(w)\right|$. If $f^{n}(z) \notin \mathbb{R}$ then, using this inequality for $w=$ $f(z), \ldots, f^{n-1}(z)$, we obtain

$$
\frac{\left|\operatorname{Im}\left(f^{n}(z)\right)\right|}{|\operatorname{Im}(f(z))|}=\prod_{k=1}^{n-1} \frac{\left|\operatorname{Im}\left(f\left(f^{k}(z)\right)\right)\right|}{\left|\operatorname{Im}\left(f^{k}(z)\right)\right|} \leq \prod_{k=1}^{n-1}\left|f^{\prime}\left(f^{k}(z)\right)\right| .
$$

But $|\operatorname{Im}(f(z))| \leq|f(z)|=\left|f^{\prime}(z)\right|$, and hence

$$
\left|\operatorname{Im}\left(f^{n}(z)\right)\right| \leq \prod_{k=0}^{n-1}\left|f^{\prime}\left(f^{k}(z)\right)\right|=\left|\left(f^{n}\right)^{\prime}(z)\right| .
$$

If $f^{n}(z) \in \mathbb{R}$ then the inequality is obvious.

† Address for correspondence: Dr Michal Misiurewicz, Mathematics Institute, Warsaw University, PKiNIXp, 00-901 Warszawa, Poland. 
Let $S=\left\{z \in \mathbb{C}:|\operatorname{Im} z| \leq \frac{1}{3} \pi\right\}$.

LEMMA 2. (a) If $z \in S$ then $\operatorname{Re}(f(z)) \geq \operatorname{Re} z+(1-\ln 2)$.

(b) If $z \in \mathbb{C} \mid \mathbb{R}$ then there exists $n \geq 0$ such that $f^{n}(z) \notin S$.

Proof. For $y \in\left[-\frac{1}{3} \pi, \frac{1}{3} \pi\right]$ we have $\cos y \geq \frac{1}{2}$ and hence if $x+i y \in S$ then $\operatorname{Re}(f(x+i y))=e^{x} \cos y \geq \frac{1}{2} e^{x}$. Let $\phi(x)=\frac{1}{2} e^{x}-x$. Then $\phi^{\prime}(x)=\frac{1}{2} e^{x}-1$ and hence $\inf _{\mathbb{R}} \phi=\phi(\ln 2)=1-\ln 2$. Therefore, if $z \in S$ then $\operatorname{Re}(f(z)) \geq \operatorname{Re} z+(1-\ln 2)$.

Suppose now that $z \in \mathbb{C} \mid \mathbb{R}$ and $f^{k}(z) \in S$ for all $k \geq 0$. Since $1-\ln 2>0$, by induction we obtain $\operatorname{Re}\left(f^{k}(z)\right) \rightarrow \infty$ as $k \rightarrow \infty$. For all $y \in\left[-\frac{1}{2} \pi, \frac{1}{2} \pi\right]$ we have $|\sin y| \geq$ $(2 / \pi)|y|$. Thus

$$
\left|\operatorname{Im}\left(f^{k+1}(z)\right)\right| \geq e^{\operatorname{Re}\left(f^{k}(z)\right)} \cdot(2 / \pi)\left|\operatorname{Im}\left(f^{k}(z)\right)\right| \quad \text { for all } k \geq 0 .
$$

But for $k$ sufficiently large, $e^{\operatorname{Re}\left(f^{k}(z)\right)} \cdot 2 / \pi>2$, and hence $\left|\operatorname{Im}\left(f^{k}(z)\right)\right| \rightarrow \infty$ as $k \rightarrow \infty$ - a contradiction.

LEMMA 3. Let $B \subset \mathbb{C}$ be a disk with a centre $b$ and a radius $r$. Let $n \geq 0$ be such that $\left.f^{n}\right|_{B}$ is a homeomorphism. Then $f^{n}(B)$ contains a disk with a centre $f^{n}(b)$ and a radius $r \cdot \inf _{B}\left|\left(f^{n}\right)^{\prime}\right|$.

Proof. Let $\gamma$ be the shortest curve joining $f^{n}(b)$ with the boundary of $f^{n}(B)$. Then the curve $\left(\left.f^{n}\right|_{B}\right)^{-1}(\gamma)$ joins $b$ with the boundary of $B$. Thus its length is at least $r$. Since $f^{n}$ is holomorphic, it is conformal, i.e. stretching in all directions is equal to the absolute value of the derivative. Hence, the length of $\gamma$ is at least $r \cdot \inf _{B}\left|\left(f^{n}\right)^{\prime}\right|$.

LEMMA 4. Let $V$ be a non-empty open connected set. Then only finitely many of its images can be disjoint from $S$.

Proof. Suppose that there exists an increasing sequence $\left(n_{j}\right)_{i=1}^{\infty}$ such that $f^{n_{i}}(V)$ is disjoint from $S$ for every $j$. Then, by lemma $1, \underset{V}{\inf }\left|\left(f^{n_{i}}\right)^{\prime}\right| \geq\left(\frac{1}{3} \pi\right)^{i}$ for all $j$. If all maps $\left.f^{n_{i}}\right|_{V}$ are homeomorphisms then, by lemma 3 , there exists $k$ such that $f^{k}(V)$ contains a disk of radius $\pi$. Then there exists an integer $m$ such that $f^{k}(V)$ intersects the line $\mathbb{R}+2 \pi i m$. If some $\left.f^{n_{i}}\right|_{V}$ is not a homeomorphism then, since $V$ is connected, there exist integers $k \in\left[0, n_{j}-1\right]$ and $m$ such that $f^{k}(V)$ intersects the line $\mathbb{R}+2 \pi i m$. In both cases, $f^{k+1}(V)$ intersects the real axis. Therefore all sets $f^{n_{i}}(V)$ for $n_{j} \geq k+1$ intersect the real axis - a contradiction.

LEMMA 5. Let $V$ be a non-empty open connected set such that infinitely many of its images are contained in the half-plane $H=\{z: \operatorname{Re} z>4\}$. Then some image of $V$ intersects the real axis.

Proof. Suppose that no image of $V$ intersects the real axis. Then no image of $V$ intersects the boundary of the set $W=\{z:|\operatorname{Im} z| \leq 2 \pi$ and $|\operatorname{Im}(f(z))| \leq 2 \pi\}$. If a connected set $\boldsymbol{A}$ is disjoint from $W$ then either $\boldsymbol{A}$ or $f(A)$ is disjoint from $S$. Therefore, in view of lemma 4, only a finite number of images of $V$ can be disjoint from $W$. Hence, almost all images of $V$ are contained in $W$. If

$$
|\operatorname{Im} z|=\frac{1}{3} \pi \text { and } \operatorname{Re} z \geq 4
$$


then

$$
|\operatorname{Im}(f(z))|=e^{R e z} \sin |\operatorname{Im} z| \geq e^{4} \sin \frac{1}{3} \pi>2^{4} \cdot \frac{1}{2}=2 \cdot 4>2 \pi .
$$

Therefore the boundary of $S$ is disjoint from $W \cap H$. Thus every connected subset of $W \cap H$ is either contained in $S$ or is disjoint from $S$.

Infinitely many images of $V$ are contained in $W \cap H$. From lemma $2(a)$ it follows that $f(S \cap H) \subset H$. Therefore, in view of lemma $2(b)$, infinitely many images of $V$ are contained in $H \backslash S$. This contradicts lemma 4.

LEMMA 6. Let $V$ be a non-empty open connected set. Then some image of $V$ intersects the real axis.

Proof. Suppose that no image of $V$ intersects the real axis. By Montel's theorem, $\left\{\left.f^{n}\right|_{V}\right\}_{n=0}^{\infty}$ is a normal family of functions. By lemma 5 , almost all images of $V$ intersect the disk $D=f(\mathbb{C} \mid H)=\left\{z:|z| \leq e^{4}\right\}$. Let $f_{0}$ be a limit of some subsequence of the sequence $\left(\left.f^{n}\right|_{V}\right)_{n=0}^{\infty}$. Then $f_{0}(V)$ intersects $D$.

Take a point $z$ belonging to this intersection. If $z \in \mathbb{R}$ then there exists $k \geq 0$ such that $f^{k}(z) \in H$. If $z \notin \mathbb{R}$ then, by lemma $2(b)$, there exists $k \geq 0$ such that $f^{k}(z) \notin S$. Therefore there exists a subsequence of the sequence $\left(\left.f^{n}\right|_{V}\right)_{n=0}^{\infty}$ convergent to a map $f_{1}$ and a point $w \in V$ such that $f_{1}(w) \in H$ or $f_{1}(w) \notin S$. Then there exists a connected open neighbourhood $U$ of $w$ such that $U \subset V$ and $f^{n}(U) \subset H$ or $f^{n}(U) \cap S=\varnothing$ for infinitely many $n s$. Thus, by lemmas 4 and 5 , some image of $U$ intersects the real axis - a contradiction.

THEOREM. The set $F(f)$ is the whole plane.

Proof. Suppose that $F(f)$ is not the whole plane. Then there exists a non-empty connected open set $U$ such that $\left\{\left.f^{n}\right|_{U}\right\}_{n=0}^{\infty}$ is a normal family of functions. By lemma 6 , the set of points which are mapped eventually into $\mathbb{R}$ is dense in $U$. By lemma $2(a)$, images of all these points converge to infinity. Hence, the sequence $\left(\left.f^{n}\right|_{U}\right)_{n=0}^{\infty}$ converges uniformly to infinity. Since the set $f(\mathbb{C} \mid H)$ is bounded, almost all sets $f^{n}(U)$ are contained in $H$. Also, almost all of them intersect the real axis. By lemma 2 $(b)$, infinitely many of them are not contained in $S$. Since the boundary of $S$ is disjoint from $W \cap H$ (see the proof of lemma 5), infinitely many of the images of $U$ are not contained in $W$. Hence, infinitely many of the images of $U$ are not contained in the $\operatorname{strip}\{z:|\operatorname{Im} z| \leq 2 \pi\}$. Consequently, infinitely many of them intersect one of the lines $\mathbb{R} \pm \pi i$. But the second image of these lines is contained in the unit disk - a contradiction.

In view of Montel's theorem we obtain immediately:

Corollary. The map $f$ is topologically transitive.

This paper was written essentially during my visit to the Institut des Hautes Etudes Scientifiques (Bures-sur-Yvette, France). I gratefully acknowledge the hospitality of IHES and the financial support of Stiftung Volkswagenwerk for the visit. I am also indebted to M. Keane who told me about the problem. 


\section{REFERENCES}

[1] I. N. Baker. Limit functions and sets of non-normality in iteration theory. Ann. Acad. Sci. Fennicae, Ser. A.I. Math. 467 (1970).

[2] P. Fatou. Sur l'itération des fonctions transcendantes entières. Acta Math. 47 (1926), 337-370.

[3] H. Töpfer. Über die Iteration der ganzen transzendenten Funktionen, insbesondere von sin and cos. Math. Ann. 117 (1940), 65-84. 